Corresponding author: woodmat@ohsu.edu; davarem@ohsu.edu

(C) 2021 Keddy et al. This article is distributed under the terms of the Creative Commons Attribution-NonCommercial License, which permits reuse and redistribution, except for commercial purposes, provided that the original author and source are credited.

Ontology term: neoplasm of the central nervous system

Published by Cold Spring Harbor Laboratory Press

doi:10.1101/mcs.a006109

\section{Mechanisms of targeted therapy resistance in a pediatric glioma driven by ETV6-NTRK3 fusion}

\author{
Clare Keddy, ${ }^{1}$ Tanaya Neff, ${ }^{2}$ Jianya Huan, ${ }^{3}$ Joshua P. Nickerson, ${ }^{4}$ \\ Catherine Z. Beach, ${ }^{1}$ Yassmine Akkari, ${ }^{5}$ Jianling Ji, ${ }^{6}$ Stephen Moore, ${ }^{7}$ \\ Kellie J. Nazemi, ${ }^{1}$ Christopher L. Corless, ${ }^{2,3}$ Carol Beadling, ${ }^{2}$ Randy Woltjer, ${ }^{3}$ \\ Yoon-Jae Cho, ${ }^{1}$ Matthew D. Wood, ${ }^{2,3}$ and Monika A. Davare ${ }^{1}$ \\ ${ }^{1}$ Department of Pediatrics, Doernbecher Children's Hospital, ${ }^{2}$ Knight Diagnostic Laboratories, ${ }^{3}$ Department of \\ Pathology, ${ }^{4}$ Department of Diagnostic Radiology, Oregon Health \& Science University, Portland, Oregon \\ 97239, USA; ${ }^{5}$ Legacy Health, Department of Cytogenetics, Portland, Oregon 97209, USA; ${ }^{6}$ Department of \\ Pathology and Laboratory Medicine, Children's Hospital Los Angeles and Keck School of Medicine at \\ University of Southern California, Los Angeles, California 90033, USA; ${ }^{7}$ Department of Molecular and Medical \\ Genetics, Oregon Health \& Science University, Portland, Oregon 97239, USA
}

\begin{abstract}
Chromosomal rearrangements of the NTRK genes generate kinase fusions that are targetable oncogenic drivers in diverse adult and pediatric malignancies. Despite robust clinical response to targeted NTRK inhibition, the emergence of therapeutic resistance poses a formidable clinical challenge. Here we report the characterization of an ETV6NTRK3 fusion-driven pediatric glioma that progressed through NTRK-targeted treatments with entrectinib and selitrectinib. Genetic analysis of multifocal recurrent/resistant lesions identified a previously uncharacterized NTRK3 p.G623A and a known p.G623E resistance mutation, in addition to other alterations of potential pathogenic impact. Functional studies using heterologous reconstitution model systems and patient-derived tumor cell lines establish that NTRK3 ${ }^{\mathrm{G} 623 \mathrm{~A}}$ and NTRK3 ${ }^{\mathrm{G} 623 \mathrm{E}}$ mutated kinases exhibit reduced sensitivity to entrectinib and selitrectinib, as well as other NTRK inhibitors tested herein. In summary, this genetic analysis of multifocal recurrent/resistant glioma driven by ETV6-NTRK3 fusion captured a cross section of resistance-associated alterations that, based on in vitro analysis, likely contributed to resistance to targeted therapy and disease progression.
\end{abstract}

[Supplemental material is available for this article.]

\section{INTRODUCTION}

Gene fusion events involving the neurotrophin receptors TRKA, TRKB, and TRKC, encoded by NTRK1, NTRK2, and NTRK3, respectively, are oncogenic drivers in a diversity of adult and pediatric tumor types (Cocco et al. 2018). The neurotrophin receptor family plays a role in nervous system development, maturation, and maintenance through regulation of key intracellular signaling pathways (Huang and Reichardt 2003). Across organ systems, tumors driven by NTRK gene fusions exhibit clinically meaningful responses to targeted NTRK inhibition (Doebele et al. 2020; Hong et al. 2020), leading to approval of larotrectinib and entrectinib for the treatment of NTRK-rearranged cancers by the United States Food and Drug Administration (FDA). Primary central nervous system (CNS) tumors driven by NTRK fusions are increasingly recognized, with cases showing a remarkable range of histological patterns, 
clinical demographics, radiologic appearances, and co-occurring molecular alterations (Torre et al. 2020). Examples of primary CNS tumors responding to NTRK inhibition are reported, making this a promising molecular alteration for precision oncology strategies (Ziegler et al. 2018).

Despite robust clinical responses to targeted kinase inhibitors, the durability of therapeutic effect is frequently hampered by the emergence of acquired resistance. Elucidating the mechanisms of resistance to targeted kinase inhibitors (TKIs) in order to identify effective second-line therapeutics is thus an important area of investigations in the field. To date, reported mechanisms of clinical resistance to larotrectinib and entrectinib include kinaseintrinsic alterations that negatively affect inhibitor affinity for the kinase (Russo et al. 2016; Drilon et al. 2018a; Somwar et al. 2020) and bypass pathway alterations that compensate for loss of NTRK signaling. Previously reported larotrectinib- and entrectinib-resistant NTRK kinase domain (KD) mutations include substitutions of the glycine residue in the exposed solvent front of the kinase domain (solvent front: NTRK $1^{\mathrm{G} 595 \mathrm{R}}$ and NTRK3 ${ }^{\mathrm{G} 623 \mathrm{R}}$ ) or substitutions of the glycine residue preceding the critical aspartic acid (D)-phenyalanine (F)-glycine(G) motif in the activation loop of the kinase (xDFG: NTRK1 ${ }^{\mathrm{G} 667 \mathrm{C}}$, NTRK1 ${ }^{\mathrm{G} 667 \mathrm{~S}}$, and NTRK3 ${ }^{\text {G696A) }}$ (Drilon et al. 2016a, 2018a; Russo et al. 2016; Somwar et al. 2020). Kinase-extrinsic or bypass alterations are less extensively studied; however, MAPK pathway alterations were reported as a TKI bypass mechanism in non-CNS tumors (Cocco et al. 2019).

Rational drug development strategies to mitigate kinase-intrinsic resistance led to second-generation NTRK inhibitors (NTRKi) including selitrectinib (LOXO-195), repotrectinib (TPX-0005), and taletrectinib (Ds-6051b) (Drilon et al. 2017, 2018b; Katayama et al. 2019). Second-generation inhibitors reportedly possess preclinical efficacy against some resistant mutations (Drilon et al. 2017, 2018b; Katayama et al. 2019), including the solvent front substitutions G595R and G623R in the setting of TPM3-NTRK1 and ETV6-NTRK3 fusions, respectively. Notably, selitrectinib response was observed in a pediatric patient with recurrent infantile fibrosarcoma that harbored the ETV6-NTRK3 ${ }^{\mathrm{G} 623 \mathrm{R}}$ mutation (Drilon et al. 2017), and repotrectinib response was reported in an ETV6-NTRK3 ${ }^{\mathrm{G} 623 \mathrm{E}}$ mutant mammary analog secretory carcinoma that progressed on entrectinib (Drilon et al. 2018b).

Here, we report a case of a pediatric patient with a glioma driven by ETV6-NTRK3 fusion who developed therapeutic resistance to entrectinib and subsequently progressed through treatment intervention with a second-generation NTRK inhibitor, selitrectinib. Genetic analysis of the resistant tumors revealed NTRK $3^{\mathrm{G} 623 \mathrm{~A}}$ and NTRK3 ${ }^{\mathrm{G} 623 \mathrm{E}}$ resistant mutations. The functional impact of these kinase domain substitutions was characterized through in vitro studies, establishing these variants as contributors to NTRK targeted therapy resistance. This report demonstrates that similar resistance mechanisms for systemic NTRK fusion-driven tumors are relevant in primary CNS tumors with NTRK gene rearrangements, and testing for resistance-associated alterations could be beneficial to guide management of resistant/recurrent NTRK-related CNS neoplasia.

\section{RESULTS}

\section{Case Presentation}

A 3-yr-old female presented to an emergency department with $~ 2$ mo of morning emesis, becoming intractable and associated with headaches in recent weeks. Neuroimaging revealed extensive solid and cystic signal abnormality along the ventricular margins and extending into the left parietal lobe white matter (Fig. 1A,B), along with bilateral cerebellopontine angle masses and foci of abnormal enhancement in the spine. An excisional biopsy of the left parietal region tumor revealed an unusual biphasic spindled and epithelioid 


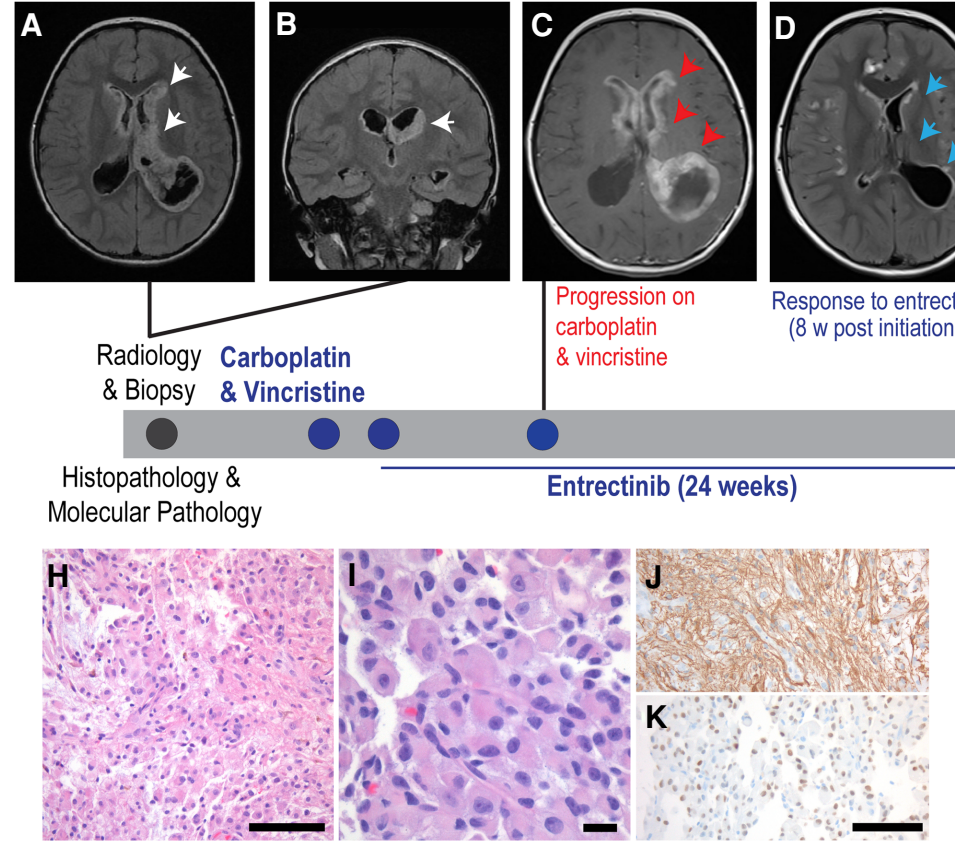

$\mathbf{L}$

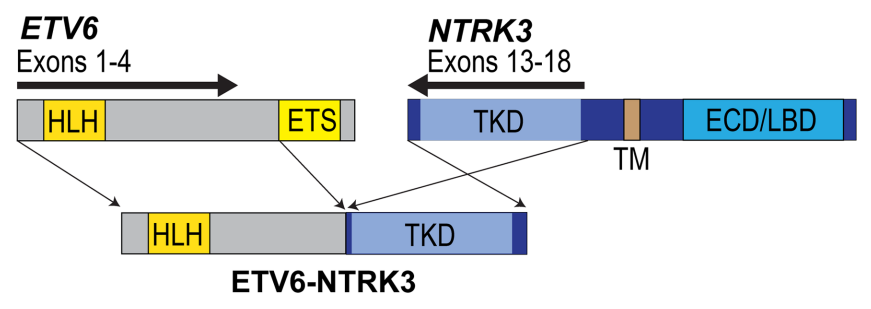

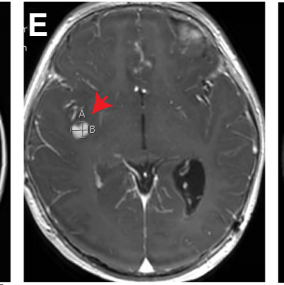

Progre

Progression on entrectinib Progression on selitrecti
(24 w post initiation)

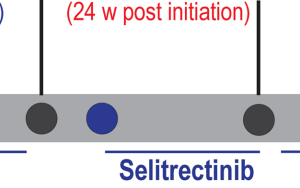

(8 weeks)

Bevacizumab,

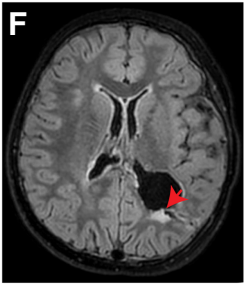

Patient deceased

14 months post diagnosis

(8 w post initiation)

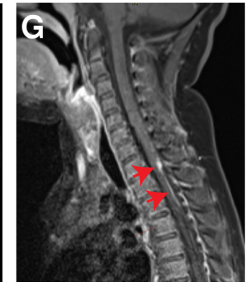

Progression on selitrectinib

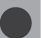

Temozolomide, Irinotecan,

Larotrectinib \&

Radiation (16 weeks)
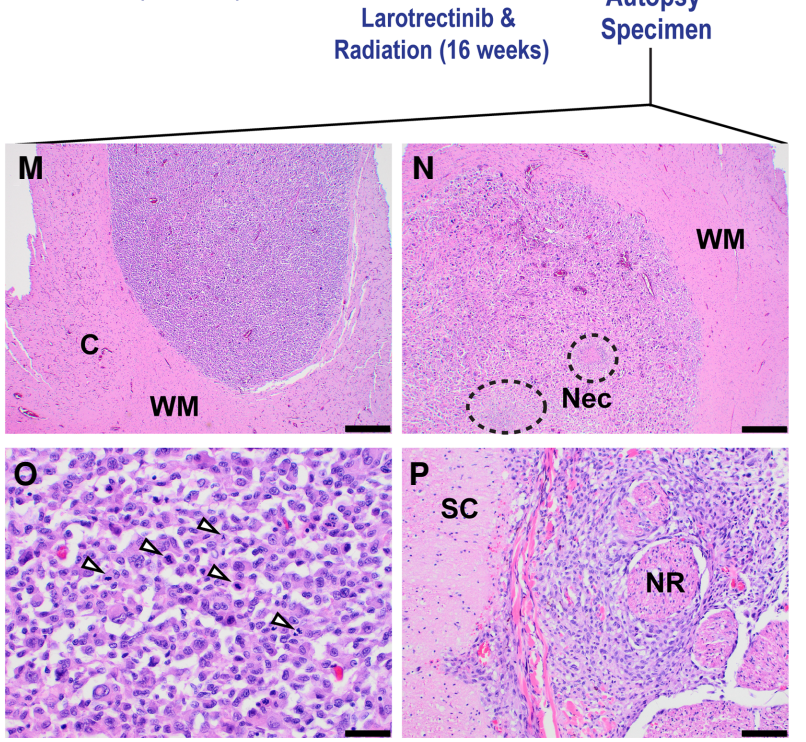

Figure 1. Clinical history and histopathological findings of the case. (A-G) Timeline showing the treatment course of the patient with corresponding radiographs indicating location and progression of tumors. Initial pretreatment T2 FLAIR axial and coronal MR images $(A, B)$ demonstrate T2 hyperintense nodular tissue along the ependymal surfaces of the lateral ventricles, greater on the left than the right. T1 post-contrast image shows evidence of progression on carboplatin plus vincristine therapy $(C)$. Following initial entrectinib therapy there is marked reduction in the volume of tumor on axial T2 FLAIR imaging (D). Post-gadolinium axial T1 images (E) reveal disease progression with new enhancing nodular tissue in the sylvian fissure. The image in panel $F$ also shows new T2 FLAIR nodularity at the ventricular margin. Cervical spine sagittal T1 post-gadolinium imaging $(G)$ revealed new leptomeningeal tumor along the dorsal surface of the cord. ( $H-K)$ Histology and immunophenotype of the diagnostic excisional biopsy at clinical presentation. Hematoxylin and eosin (H\&E)-stained sections showed a predominantly epithelioid neoplasm with spindled and myxoid areas and delicate fibrillary processes ( $H$; scale bar, 100 microns). Most of the tumor cells showed abundant eosinophilic cytoplasm, moderately pleomorphic nuclei, and conspicuous nucleoli (l; scale bar, 20 microns). Immunoreactivity for glial fibrillary acidic protein (GFAP; $J$ ) and Olig-2 ( $K$; scale bar, 100 microns applies to $J$ and $K$ ). (L) Domain organization for ETV6, NTRK3, and ETV6-NTRK3 fusion. Exons 1-4 of ETV6 and exons 14-20 of NTRK3 form the final fusion product. The helix-loop-helix (HLH) of ETV6 and the tyrosine kinase domain (TKD) of NTRK3 are maintained in the ETV6-NTRK3 fusion. (M-P) H\&E stained sections of postmortem tumor samples. Nodules of cerebral hemispheric disease showed a densely cellular, markedly pleomorphic glioma with brisk mitotic activity and focal necrosis (Nec), and limited infiltration of adjacent brain cortex (C) and white matter (WM) ( $M-N$; scale bars, 500 microns). On higher magnification, frequent mitotic figures (arrowheads) were noted, with tumor cells showing high nuclear to cytoplasmic ratios and vesicular chromatin ( $N$; scale bar, 50 microns). A section of spinal leptomeningeal disease showed limited infiltration of the spinal cord (SC) parenchyma, and a coating of pleomorphic tumor encasing the spinal nerve roots (NR) ( $P$; scale bar, 100 microns). 
neoplasm that showed variable immunoreactivity for glial markers glial fibrillary acidic protein (GFAP) and Olig-2 (Fig. 1 H-K). Mitotic figures were present at up to two mitotic figures per 10 high-power fields, whereas necrosis and microvascular proliferation were not identified. A clinical next-generation sequencing study on the Children's Hospital of Los Angeles OncoKids panel identified a fusion between ETV6 and NTRK3 predicted to generate fusion protein composed of exons 1-4 of ETV6 and exons 14-20 of NTRK3, a region that includes most of the NTRK3/TRKC kinase domain (Fig. 1L). No pathogenic mutations were identified in other assessed genes (Hiemenz et al. 2018). Copy-number profiling by single-nucleotide polymorphism microarray showed focal homozygous deletion of 9p21, encompassing the $C D K N 2 A / B$ tumor suppressor gene, and numerous whole-chromosome losses (Supplemental Fig. S1A). The patient was initially treated with carboplatin and vincristine per Children's Oncology Group protocol A9952 regimen A for 4 wk, and then transitioned to entrectinib (300 $\mathrm{mg}$ daily) because of early clinical and radiologic progression. Dramatic clinical and radiographic improvement was seen after 8 wk of therapy (Fig. 1C, D). Entrectinib response persisted for a total of $24 \mathrm{wk}$, when treatment was discontinued because of tumor progression (Fig. 1E). The patient transitioned to a second-generation NTRK inhibitor, selitrectinib (LOXO-195), with early radiographic recurrence/progression after 8 wk of treatment (Fig. 1F). Subsequent therapies included a combination regimen of larotrectinib, irinotecan, bevacizumab, and temozolomide and focal palliative radiation therapy to midbrain and hypothalamic lesions. Radiographic monitoring of late disease showed numerous cortically based and periventricular nodular lesions and extensive tumor encasement of the spinal cord and spinal nerve roots (Fig. 1G). The patient succumbed to disease while receiving hospice care 14 mo after initial diagnosis. An autopsy limited to the brain and spinal cord was performed.

\section{Genomic Profiling of Recurrent/Disseminated Disease after NTRK Inhibitor Treatment}

On gross examination of the brain, innumerable nodules of recurrent/disseminated tumor were identified along the ventricular walls. The thalamus, hypothalamus, midbrain, and pons were enlarged and firm. The spinal cord appeared enlarged, because of diffuse coating of the cord and nerve roots by confluent disseminated tumor. Three representative nodular lesions from the cerebral hemispheres and one region of spinal leptomeningeal disease were sampled and showed a pleomorphic, mitotically active glioma with necrosis and parenchymal infiltration along perivascular spaces (Fig. 1M-P). The samples were submitted for GeneTrails, a next-generation sequencing panel that covers mutations and small insertions/deletions in 222 cancer-related genes as well as fusion coverage for 24 genes including NTRK1/2/3. Gene coverage for DNA sequencing and RNA fusion panel is presented in Supplemental Tables 1 and 2, respectively, and sequencing coverage for RNA fusion panel is in Supplemental Table 3. Extracted nucleic acids from the original clinical sample were resequenced on the same panel to help identify mutations associated with recurrent/progressive disease. The pathogenic ETV6-NTRK3 fusion was detected in all four samples (Table 1; Fig. 2A). The sample from disseminated spinal leptomeningeal disease revealed an acquired uncharacterized NTRK3 p.G623A kinase domain mutation (Table 1). Two cerebral lesion showed a NTRK3 p.G623E mutation, known to be associated with NTRK inhibitor resistance, along with multiple acquired mutations with a preponderance of $G: C$ to $A: T$ transitions compatible with sequelae of alkylating agent chemotherapy (Table 1; Fig. 2A; Supplemental Fig. S2; Drilon et al. 2018b). The samples with NTRK3 resistance mutations all showed a shared, acquired, pathogenic mutation TP53 p.R282W, a well-known inactivating mutation impacting p53 protein function (ClinVar ID: 12364). Intriguingly, the final cerebral hemispheric focus with confirmed ETV6-NTRK3 rearrangement showed neither NTRK3 


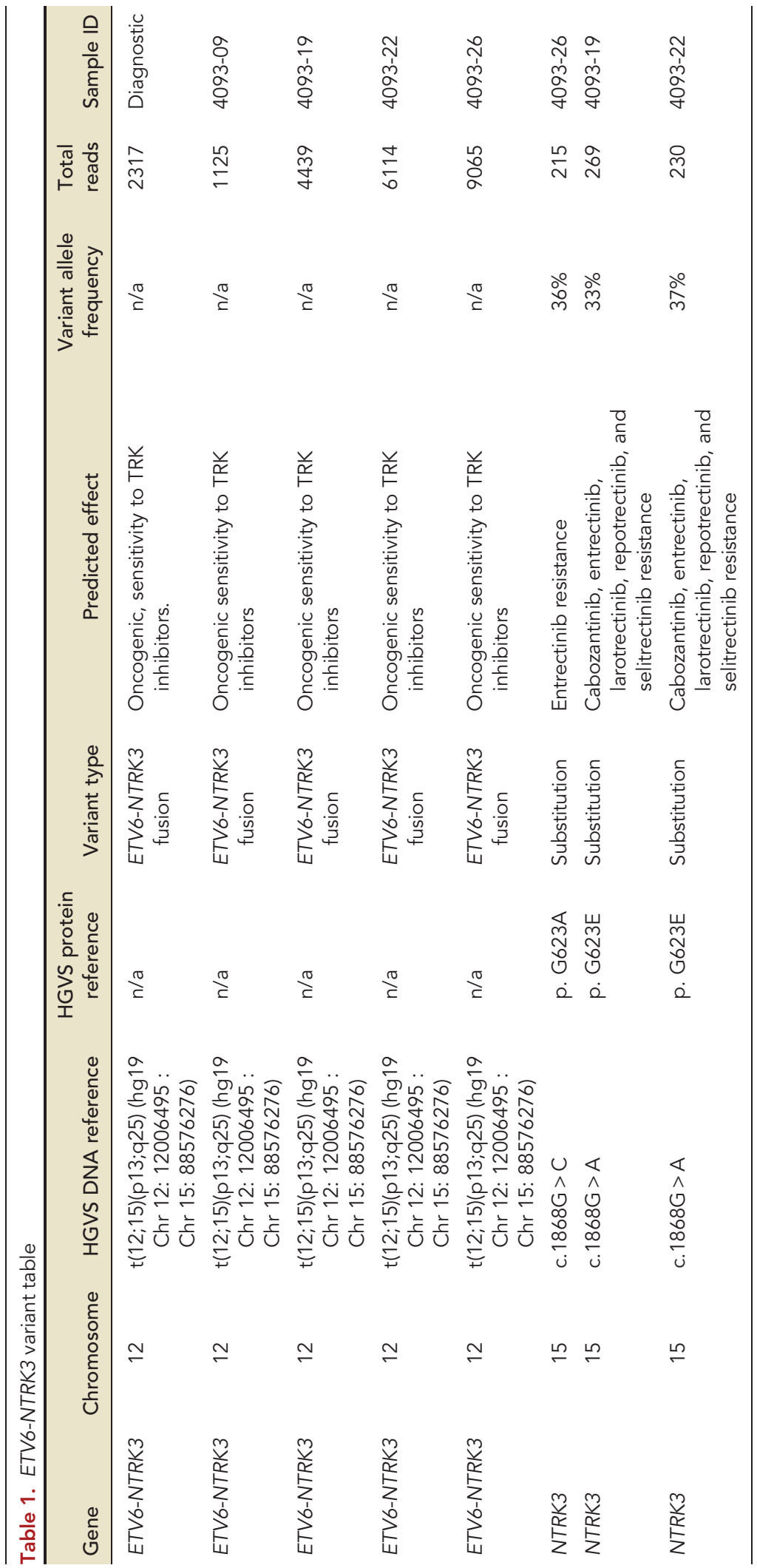


A

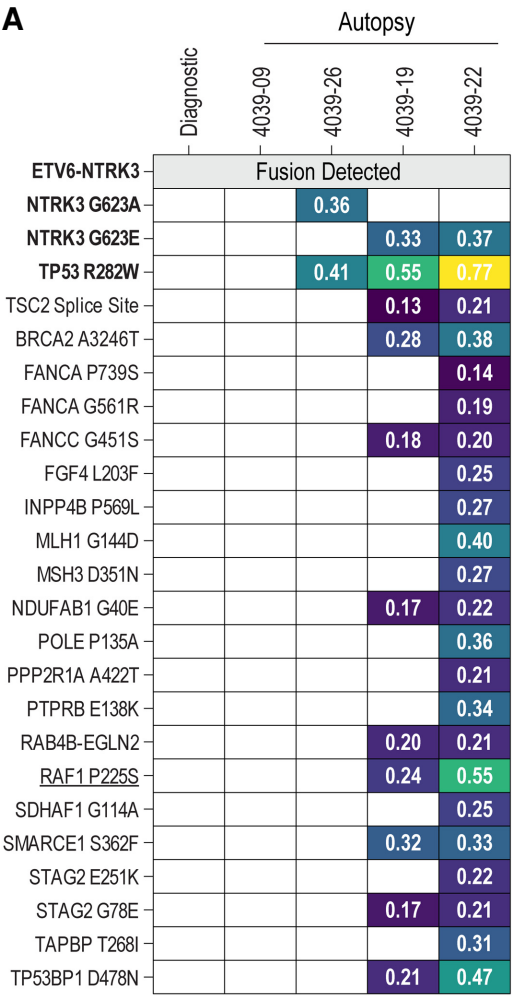

B
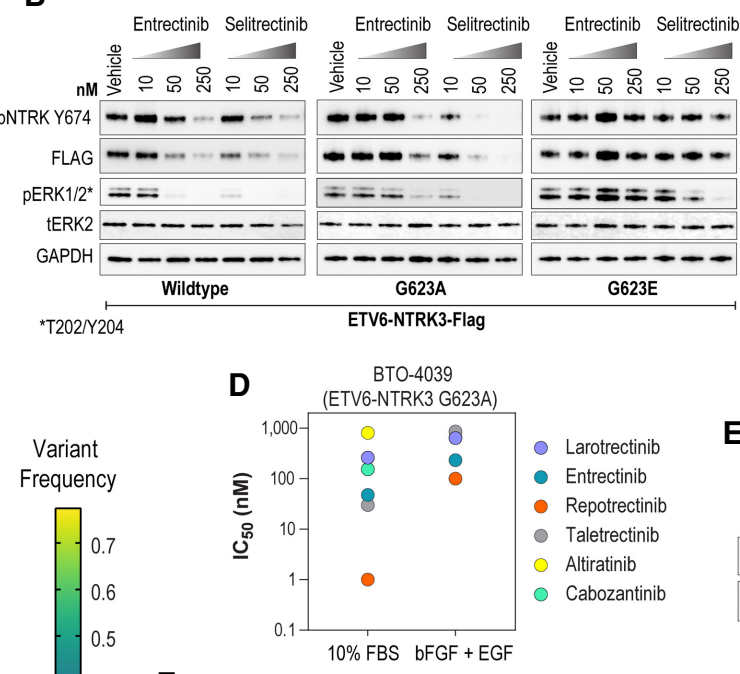

$\mathbf{F}$

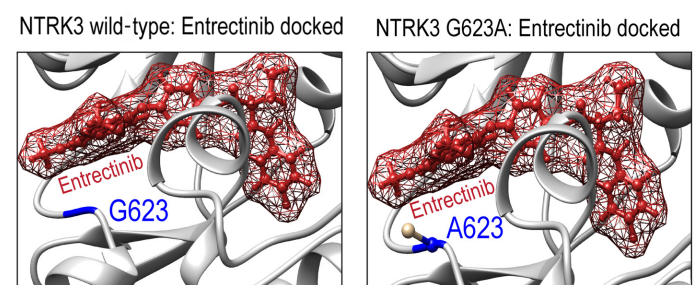

C

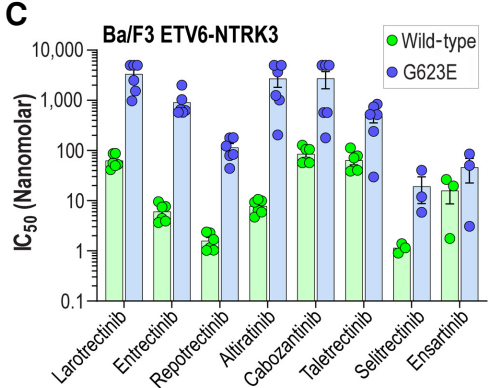

E

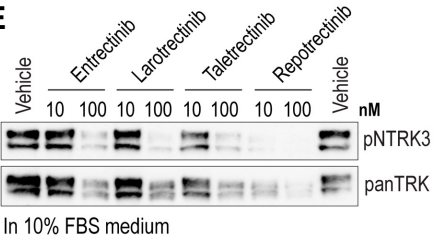

NTRK3 G623E: Entrectinib docked

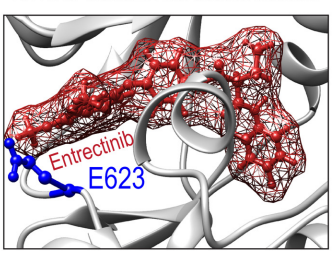

Figure 2. Genetic analysis of tumors and in vitro characterization of identified NTRK3 mutations. (A) Variant allele frequency of identified mutations from GeneTrails next-generation sequencing of diagnostic and autopsy samples. (B) Immunoblot analysis of phosphorylated NTRK3, flag-tagged NTRK3, and phosphorylated and total ERK from transfected HEK293T17 cell lysates after $4 \mathrm{~h}$ of treatment with TKIs as indicated. (C) Bar graph of cell proliferation $\mathrm{IC}_{50}$ values for each of the indicated TKIs against $\mathrm{Ba} / \mathrm{F} 3$ cells expressing wild-type ETV6NTRK3 (green) and ETV6-NTRK3 ${ }^{\text {G623E }}$ (blue). (D) Scatter plot of cell proliferation IC 50 values for indicated TKls against patient cell line BTO-4039 expressing ETV6-NTRK3 ${ }^{\text {G623A }}$ cultured in 10\% FBS media or neural stem cell media supplemented with bFGF and EGF. (E) Immunoblot analysis of phosphorylated NTRK3 and total NTRK3 from patient cell line BTO-4039 after $5 \mathrm{~h}$ of treatment with TKls as indicated. (F) Predicted binding poses for entrectinib with NTRK3 ${ }^{\mathrm{WT}}$, NTRK3 ${ }^{\mathrm{G} 623 \mathrm{~A}}$, and NTRK3 ${ }^{\mathrm{G} 623 \mathrm{E}}$

resistance mutation nor TP53 mutation, even though it was histologically similar to the other foci of disease with resistance mutations, with marked pleomorphism and mitotic activity. The complete genetic findings are listed in Supplemental Table 4, and summarized schematically along with variant allele frequencies in Figure 2A.

\section{Solvent Front NTRK3 G623A/E Mutation Confers Broad Resistance to NTRK Inhibitors}

To determine the functional significance of the NTRK3 ${ }^{\mathrm{G} 623 \mathrm{~A}}$ and NTRK $3^{\mathrm{G} 623 \mathrm{E}}$ variants as contributors to TKI resistance, these amino acid substitutions were engineered into an ETV6NTRK3-FLAG construct and their impact on NTRK autophosphorylation and signaling effector pathway activation with or without NTRKi treatment was examined. Immunoblotting of cell lysates shows entrectinib and selitrectinib inhibit ETV6-NTRK3 autophosphorylation and downstream activation of MAPKs, ERK1/2. Compared to ETV6-NTRK3WT, ETV6NTRK3 ${ }^{\mathrm{G} 623 \mathrm{~A}}$ confers partial resistance to entrectinib that is particularly evident in the lack of inhibition of MAPK signaling (ERK1/2 phosphorylation) at a $50 \mathrm{nM}$ dose (Fig. 2B). ETV6NTRK3 ${ }^{\text {G623E }}$ is completely resistant to entrectinib in these assays, with no inhibition of 
autophosphorylation or MAPK signaling noted even at $250 \mathrm{nM}$ treatment and is less sensitive to selitrectinib as compared to ETV6-NTRK3WT.

To further explore the TKI resistance profile of NTRK3 ${ }^{\mathrm{G} 623 \mathrm{E}}$ mutation, we used the $\mathrm{Ba} / \mathrm{F} 3$ heterologous model system (Warmuth et al. 2007; Davare et al. 2015; Drilon et al. 2016b; Somwar et al. 2020). Stably transformed Ba/F3 ETV6-NTRK3 ${ }^{\mathrm{WT}}$ and ETV6-NTRK3 ${ }^{\mathrm{G} 623 \mathrm{E}}$ cell lines were used for dose-response cell viability assays with a panel of NTRKi. Among these, altiratinib and cabozantinib are Type II inhibitors binding the DFG-out conformation, whereas the others are Type I inhibitors binding the DFG-in conformation. ETV6-NTRK3 ${ }^{\text {WT }}$ and ETV6-NTRK3 ${ }^{\text {G623E }}$ harbor comparable transformative propensity in IL-3 withdrawal assays (Supplemental Fig. S1B). The NTRK3 ${ }^{\mathrm{G} 623 \mathrm{E}}$ mutation has a significant impact on the concentration required to inhibit cell growth by $50 \%\left(\mathrm{IC}_{50}\right)$ for larotrectinib (53-fold increase), entrectinib (148-fold increase), repotrectinib (71-fold increase), altiratinib (344-fold increase), and cabozantinib (32-fold increase). The inhibitory efficacy of taletrectinib, selitrectinib, and ensartinib is also blunted by the NTRK3 ${ }^{\mathrm{G} 623 \mathrm{E}}$ mutation with approximately eightfold, 17fold, and approximately threefold increases in $\mathrm{IC}_{50} \mathrm{~S}$, respectively, as compared to the wild-type (Fig. 2C; Supplemental Fig. S3).

Mutations of RAS/RAF/MEK pathway genes that constitutively activate the MAPK pathway were reported as a recurrent mechanism of resistance to NTRKi in NTRK fusion-positive cancers (Cocco et al. 2019). Given this, we were intrigued by our identification of a RAF1 P225S variant of unknown significance present in two of the autopsy tumor samples (Fig. 2A). RAF kinases transmit signal from RAS proteins to downstream MAPK proteins. Of note, RAF1 ${ }^{\text {P225S }}$ resides in a loop connecting the RAF1 RAS-binding and kinase domains (Supplemental Fig. $\mathrm{S} 4 \mathrm{~A})$. Another nearby mutation in this loop region, RAF1 ${ }^{\mathrm{P} 261 \mathrm{~S}}$, is well-established as a gainof-function mutation in cancer (Noeparast et al. 2019) and as a germline mutation implicated in RASopathies including Noonan syndrome and LEOPARD syndrome (Pandit et al. 2007). We examined the functional impact of RAF1 ${ }^{\text {P225S }}$ relative to RAF1 ${ }^{\mathrm{WT}}$ and known activating $\mathrm{RAF1}^{\mathrm{P} 261 \mathrm{~S}}$ as a positive control. Transfection of these constructs into HEK293A cells establish that the RAF1 ${ }^{\text {P225S }}$ variant is a functionally neutral substitution with activity comparable to RAF $1^{\text {WT }}$ as compared to the robust activation of phospho-ERK1/2 by RAF1 ${ }^{\text {P261S }}$, supporting that RAF1 ${ }^{\text {P225S }}$ was not a contributing factor to NTRKi resistance noted in these tumor samples (Supplemental Fig. S4B).

A patient-derived cell line was established from one of the autopsy tissue samples (BTO-4039-26, Supplemental Fig. S1C). Sanger sequencing of cDNA confirmed the expression of ETV6-NTRK3 fusion product and showed the presence of the ETV6NTRK3 ${ }^{\text {G623A }}$ mutation (Supplemental Fig. S1D). BTO-4039-26 was cultured in neural stem cell media containing supplementation with recombinant human epidermal growth factor (rh-EGF) and basic fibroblast growth factor (rh-bFGF) and also adapted to grow in DMEM with $10 \%$ FBS (D10-FBS). Dose-response cell viability assays of cells cultured in D10-FBS show higher sensitivity to inhibitors as compared with culture in neural stem cell media (Fig. 2D; Supplemental Fig. S5), suggesting that in the presence of growth factors, the tumor would show an even more limited response to targeted inhibitors (Fig. 2D; Supplemental Fig. S5). Immunoblotting of cell lysates prepared from BTO-4039-26 following treatment with select NTRKi shows that at the higher concentration (100 nM) tested, the inhibitors exhibit partial efficacy to block ETV6-NTRK3 autophosphorylation (Fig. 2E). These data are consistent with the partial resistance of ETV6-NTRK3 ${ }^{\mathrm{G} 623 \mathrm{~A}}$ seen in the transient transfection experiments.

To gain insight on the structural impact of these G623A and G623E substitutions on entrectinib binding, we docked entrectinib in the NTRK3 kinase domain using a previously generated computational homology model ${ }^{7}$ (Fig. 2F; Supplemental Fig. S6). Docking studies suggest that steric hindrance generated from the larger side chains of alanine, and particularly of glutamic acid, as compared to the native glycine at the 623 position, likely 
interferes with entrectinib binding. This offers a structural rationale for entrectinib resistance of NTRK3 ${ }^{\mathrm{G} 623 \mathrm{~A}}$ and NTRK3 ${ }^{\mathrm{G} 623 \mathrm{E}}$.

\section{DISCUSSION}

Although targeted therapeutics are becoming increasingly available, therapeutic resistance is an inevitable issue. Here, we present a case of primary ETV6-NTRK3 fusion-driven pediatric glioma with progression on multiple targeted NTRK inhibitors. This analysis identified solvent front mutations in the tyrosine kinase domain of NTRK3, similar to previously identified resistance mechanisms in NTRK1 (G595R) and NTRK3 (G623R) in extracranial NTRK fusion-driven neoplasia (Drilon et al. 2016a, 2017, 2018a,b; Russo et al. 2016; Hemming et al. 2020). This case report demonstrates the clinical relevance of these mechanisms of resistance in patients with primary CNS malignancies that, to our knowledge, have yet to be reported. Notably, this case also demonstrates that a next-generation inhibitor such as selitrectinib, which was previously shown to have efficacy in ETV6-NTRK3 ${ }^{\mathrm{G} 623 \mathrm{E}}$-driven extracranial tumors, may have limited benefit in primary CNS tumors, potentially because of lower achievable CNS concentration.

Of the mutations found in the autopsy samples, relatively few are definitively pathogenic. TP53 ${ }^{\mathrm{R} 282 \mathrm{~W}}$ identified in three postmortem samples is a known hotspot in human cancers affecting the DNA binding ability of p53 (Supplemental Table 4; Xu et al. 2011; Chang et al. 2016; Gao et al. 2017). The TSC2 splice-site variant identified in two postmortem samples is likely to be pathogenic, as splice variants of TSC2 are reported in a variety of cancers to have potential impact on activity (Cerami et al. 2012; Gao et al. 2013). The majority of the mutations are variants of uncertain significance with unknown impact on protein function or disease pathology. Here we showed that the RAF1 ${ }^{\mathrm{P} 225 \mathrm{~S}}$ is a neutral impact mutation, unlikely to contribute to drug resistance or disease progression. POLE ${ }^{\mathrm{P} 135 \mathrm{~A}}$ has not been previously reported, but POLE ${ }^{\mathrm{P} 135 \mathrm{~S}}$ mutation was suggested to be a nonpathogenic passenger mutation (Campbell et al. 2017). From a structural perspective, we postulate that the alanine (A) and serine (S) amino acids have similar bulk and are not charged; thus, it is unlikely that P135A would impact function if $\mathrm{P} 135 \mathrm{~S}$ does not. Of the remaining mutations, many have been previously identified in patients, but functional impacts have not been elucidated (Cerami et al. 2012; Gao et al. 2013).

Although NTRK3 ${ }^{\mathrm{G} 623 \mathrm{~A}}$ and $\mathrm{NTRK}^{\mathrm{G} 623 \mathrm{E}}$ were not detected in the original clinical sample, we cannot exclude the possibility of a minor preexisting population of tumor cells harboring these alterations that was below the threshold of detection of these NGS assays. Regardless of whether NTRK3 ${ }^{\mathrm{G} 623 \mathrm{~A}}$ or NTRK3 ${ }^{\mathrm{G} 623 \mathrm{E}}$ represent a preexisting subclone or de novo acquired mutation, their reduced affinity for NTRKi was most likely permissive to their selection and expansion in the presence of inhibitor, thus contributing to disease progression. The NTRK $3^{\mathrm{G} 623 \mathrm{R}}$ solvent front substitution has been shown to be sensitive to selitrectinib, both in model systems and patients (Drilon et al. 2017). Specifically, Drilon et al. (2017) demonstrated that selitrectinib treatment induced rapid tumor response and extended disease control in a larotrectinib-resistant ETV6-NTRK3 ${ }^{\mathrm{G} 623 \mathrm{R}}$ fusion-positive infantile fibrosarcoma. In contrast to this, the ETV6-NTRK3 ${ }^{\text {G623A }}$ and ETV6-NTRK3 ${ }^{\text {G623E }}$ lesions progressed during the 8 wk of selitrectinib treatment (ClinicalTrials.gov identifier: NCT03215511 [43 mg/m²]). $\mathrm{Ba} / \mathrm{F} 3$ cell model-based studies show that ETV6-NTRK3 ${ }^{\mathrm{G} 623 \mathrm{E}}$ exhibits $\sim 18$-fold increase in $\mathrm{IC}_{50}$ for selitrectinib. We postulate that achievable CNS concentrations of selitrectinib with the pediatric dosing regimen may have been insufficient to control tumor cells harboring the kinase domain mutant ETV6-NTRK3 fusion protein that required 18-fold higher inhibitor amounts compared to wild-type fusion in our model cell system. It is also possible that the 
trophic microenvironment of the pediatric brain confers exogenous resistance, dampening the tumor response by activating basal growth or survival pathways in these cells. Indeed the patient derived cell line is highly resistant to the NTRKi when cultured with recombinant human EGF or bFGF, as opposed to generic media with fetal bovine serum (Fig. 2D).

Genetic analyses of multiple postmortem tumors showed variation in mutational landscape across distinct disseminated sites of disease in the CNS. These postmortem samples were collected after progression on entrectinib, selitrectinib, and a combination regimen that included larotrectinib and temozolomide. In the postmortem sample with the highest somatic mutation burden, $>95 \%$ of them are G:C to A:T transitions, a pattern consistent with temozolomide-associated mutation (Supplemental Fig. S2; Johnson et al. 2014; Degasperi et al. 2020). Interestingly, one of the autopsy samples (4039-09) showed no additional alterations as compared to the original clinical sample. Despite first- and second-generation NTRK inhibitor treatments, this tumor lesion grew and formed a persistent, viable nodule. This suggests several possibilities. Although NTRKi are cytotoxic in NTRK fusiondriven in vitro model systems, their effect in vivo may be cytostatic rather than cytotoxic because of the pharmacokinetics in the CNS and the brain microenvironment. It is also possible that alterations in genes not included in our NGS panel are responsible for therapeutic resistance in 4039-09 tumor.

Based on functional data presented here, we conclude that NTRK3 ${ }^{\mathrm{G} 623 \mathrm{E}}$ and, to a lesser degree, NTRK3 ${ }^{\mathrm{G} 623 \mathrm{~A}}$ were the causative mechanisms of targeted therapeutic resistance in this tumor. Furthermore, these alterations are found to confer cross-resistance to essentially all current NTRK inhibitors. Solvent front mutations have previously been observed in NTRK fusion-driven cancers, but this case represents the first report of these mutations in a primary CNS tumor. Further studies are needed to continue to assemble the profile of NTRK mutations that exhibit resistance to TRK inhibitors as well as NTRK-extrinsic mechanisms of resistance. Genetic profiling of residual and recurrent disease can shed light on these resistance mechanisms and potentially impact treatment decisions in this challenging and unusual type of glioma.

\section{METHODS}

\section{Inhibitors}

Selitrectinib (LOXO-195) and repotrectinib (TPX-0005) were purchased from MedChemExpress. Entrectinib (RXDX-101), altiratinib (DCC-2701), cabozantinib (XL184), ensartinib (X-396), larotrectinib (LOXO-101), and taletrectinib (DS-6051b) were purchased from Selleck Chemicals.

\section{Cell Culture}

Parental Ba/F3 cells (American Type Culture Collection) were cultured in complete medium (RPMI medium 1640 with 10\% fetal bovine serum [FBS], 2 mmol/L L-glutamine, penicillin, streptomycin, and fungizome) supplemented with $2 \mathrm{ng} / \mathrm{mL}$ recombinant murine IL-3 and maintained at densities of $0.5 \times 10^{6}$ to $1.5 \times 10^{6}$. HEK293A and HEK293T were cultured in complete medium (DMEM with $10 \%$ bovine growth serum, 2 mmol/L L-glutamine, penicillin, streptomycin). All cell lines were grown under standard mammalian cell culture conditions $\left(37^{\circ} \mathrm{C}, 5 \% \mathrm{CO}_{2}\right)$.

\section{$\mathrm{Ba} / \mathrm{F} 3$ Cell Line Generation}

The ETV6-NTRK3 mutants were generated using site-directed mutagenesis following manufacturer's protocol (Agilent). Platinum-E cells (Cell Biolabs, Inc.) were transfected with pCX4 
ETV6-NTRK3 wild-type or mutant constructs using DNA transfection reagent from Biotool to generate replication incompetent ecotropic retrovirus. Ba/F3 cells were infected with retrovirus encoding wild-type or mutant versions of ETV6-NTRK3. Cells were selected with $2 \mu \mathrm{g} /$ $\mathrm{mL}$ puromycin treatment. Selected Ba/F3 cells were washed three times with IL-3 free complete medium and suspended at a density of $0.5 \times 10^{6}$ per $\mathrm{ml}$. The number of viable cells were counted every other day and expanded as needed to maintain a density of $0.5 \times 10^{6}$ per $\mathrm{mL}$. Cells that grew out after IL-3 withdrawal were maintained in complete medium without IL-3 and used for in vitro assays.

\section{Sanger Sequencing Validation of Endogenous NTRK3 Mutation}

RNA was isolated using the Quick-RNA Miniprep kit (Zymo Research). cDNA was synthesized using OneScript Plus cDNA Synthesis kit (Applied Biological Materials Inc.) according to manufacturer's specifications. The NTRK3 kinase domain was polymerase chain reaction (PCR)-amplified using the primers ETV6F2 (5'-CTTCCACCCTGGAAACTCTATAC-3') and NTRKR (5'-GTCCTCCCACCCTGTAATAATC-3') and sequenced using ETV6F2. Chromatographs were aligned to identify mutations using Benchling software.

\section{DNA Transfections}

HEK293A and HEK293T cells were transiently transfected using TransIT-LT1 transfection reagent (Mirus Bio) to manufacturer's specification.

\section{Patient-Derived Tumor Cell Line}

BTO-4039-26 cells were derived from an autopsy specimen. Harvested tissue was washed with sterile saline buffer, minced under aseptic conditions, and transferred to neural stem cell medium (1:1 DMEM/F12 and neural stem cell media [ThermoFisher] with $20 \mathrm{ng} / \mathrm{mL}$ EGF, $20 \mathrm{ng} / \mathrm{mL} F G F, 1 \mathrm{ng} / \mathrm{mL} \mathrm{LIF}, 20 \mu \mathrm{g} / \mathrm{mL}$ heparin, penicillin, streptomycin, $0.025 \mathrm{M}$ HEPES, 1× B27 Supplement without A [ThermoFisher], 1× GlutaMAX [ThermoFisher], 1× Non-Essential Amino Acids Solution [ThermoFisher]). The cells were maintained in neural stem cell media. For adaptation to DMEM $+10 \%$ fetal bovine serum medium, $1 \times 10^{6}$ cells were pelleted and without rinsing, transferred to new flask containing $15 \mathrm{~mL}$ of DMEM with $10 \%$ fetal bovine serum, $2 \mathrm{mmol} / \mathrm{L}$ L-glutamine, penicillin, and streptomycin. The cells were fed with $5 \mathrm{~mL}$ of DMEM $+10 \%$ FBS once a week and cultured for no more than three population doublings in this medium.

\section{Immunoblot Analysis}

Transfected HEK293T and patient-derived cell lines were treated with the indicated concentrations of inhibitors for $4 \mathrm{~h}$. Following treatment, cells were pelleted, washed once in icecold PBS, and lysed in cell lysis buffer supplemented with 0.25\% deoxycholate, 0.05\% SDS, and protease and phosphatase inhibitors. Protein concentrations were determined using the Pierce BCA Protein Assay (ThermoFisher Scientific). Protein was extracted with NuPAGE LDS Sample Buffer (4×) (ThermoFisher Scientific) sample buffer, supplemented with $\beta$-mercaptoethanol, for $10 \mathrm{~min}$ at $75^{\circ} \mathrm{C}$. An amount of $10-25 \mu \mathrm{g}$ of extracted lysate protein was run on 4\%-12\% Bis-Tris (Invitrogen; ThermoFisher Scientific) and transferred to nitrocellulose membranes (Prometheus). Blots were probed with antibodies specific for phospho-TRK (CST; 4621; 1:1000), pan-TRK (CST; 3266; 1:1000), phospho-p44/42 MAPK (CST; 9101; 1:1000), total ERK2 (Santa Cruz; sc-1647;1:1000), phospho-S6 (CST; 4858; 1:1000), total S6 (CST; 2216; 1:1000), GFP (Origene; TA150032; 1:5000), phospho-RAF1 (CST; 9421; 1:1000), and GAPDH (Origene; OTI2D9; 1:5000). We used the Bio-Rad Chemidoc imaging station or LI-COR Odyssey imaging system and followed manufacturer's 
COLD SPR ING HARBOR Molecular Case Studies
Inhibitor resistance in NTRK3 fusion-driven glioma recommendations for immunoblot detection with use of HRP-conjugated or IR dye secondary antibodies, respectively.

\section{Cell Viability Assays}

All inhibitors were prepared as $1 \mathrm{mM}$ stock in DMSO. Inhibitors were distributed into 384well plates that were preseeded with $25 \mu \mathrm{L}$ of complete RPMI-10\% FBS medium using a D300 Digital Dispenser (Hewlett-Packard). Ba/F3 cells expressing wild-type or mutant ETV6-NTRK3 were seeded at 1000 cells per well in $25 \mu \mathrm{L}$ onto drug plates using a Multidro Combi Reagent Dispenser (Thermo Scientific). Plates were incubated for $72 \mathrm{~h}$ at $37^{\circ} \mathrm{C}$. Viability was measured using a water-soluble tetrazolium salt (WST-8) based assay (Bimake) and absorbance $(450 \mathrm{~nm})$ was read $1 \mathrm{~h}$ after adding reagent using a BioTek Synergy 2 plate reader. Data from drug-treated wells were normalized to Vehicle $(0.05 \%$ DMSO) treated wells in Microsoft Excel. Each experiment had three replicate wells per condition and the average and SEM were used for curve fit analysis. Plotting and nonlinear regression curve fit analysis for determination of $\mathrm{IC}_{50}$ values was performed using GraphPad Prism software.

\section{Homology Model}

Chimera (Pettersen et al. 2004) software was used to generate G623A and G623E substitutions (structural editing) within a previously published (Somwar et al. 2020) homology model of NTRK3 with docked entrectinib.

\section{Mutational Signature Analysis}

Signal (https://signal.mutationalsignatures.com/) was used to analyze mutational signatures from next-generation sequencing. Human GRCh37 was used as the reference genome (Degasperi et al. 2020).

\section{Next-Generation Sequencing}

Tumor-rich regions of formalin-fixed, paraffin-embedded sections of autopsy tissue were macrodissected, deparaffinized, and total nucleic acid was extracted and purified using the Nucleospin tissue kit (Machery-Nagel). Amplicon-based sequencing libraries (custom QiaSeq panel, OIAGEN, Inc.) covering the protein-coding regions of 222 cancer-related genes (see Supplemental Table 1) were prepared from tumor nucleic acid samples and sequenced on an Illumina NextSeq550. The Knight Diagnostic Laboratories GeneTrails sequencing panel covers 222 cancer-associated genes, with a total sequencing footprint of $0.61 \mathrm{Mb}$ and average read depth of $\sim 2000$. Single-nucleotide variations and small insertions/deletions are identified using FreeBayes and MuTect2 algorithms in a custom analysis pipeline. Copy-number alterations and microsatellite instability at 227 short repeats are also assessed. An RNA-based panel covering breakpoints in 21 genes detects rearrangements with any fusion partner (see Supplemental Table 2). The ETV6-NTRK3 fusion was confirmed in each sample by next-generation sequencing of tumor RNA using a custom ampliconbased library (QiaSeq) run on an Illumina NextSeq550. The panel includes partner-agnostic coverage of common known breakpoints in NTRK3.

\section{ADDITIONAL INFORMATION}

\section{Data Availability}

Data produced for clinical diagnostic purposes will not be publicly available. Research-related data will be made available without restrictions upon request by contacting the 
Competing Interest Statement

The authors have declared no competing interest.

\section{Referees}

Theodore Nicolaides

Anonymous

Received May 25, 2021; accepted in revised form July $26,2021$. corresponding authors. The variants reported herein were submitted to ClinVar (https://www .ncbi.nlm.nih.gov/clinvar/) and can be found under accession numbers SCV001781535SCV001781538.

\section{Ethics Statement}

Studies performed on deidentified human postmortem tissue are not considered "human subjects" research and have been exempted from review by the IRB. Unrestricted consent for use of the tissue is obtained from the next of kin before autopsy.

\section{Acknowledgments}

The authors thank Qianyue Yang for her assistance with maintenance of patient-derived cell lines and Dr. Phil Stork (Oregon Health \& Science University) for generously sharing the RAF1 constructs. This work was partially supported by funding from Hero-Up for Children's Cancer.

\section{Author Contributions}

J.P.N., K.J.N., R.W., Y-J.C., and M.D.W. provided clinical care of the patient and clinical data and/or autopsy tumor specimen or other resources. C.K., J.H., C.Z.B., R.W., M.D.W., and M.A.D. performed experiments and analyzed data. M.A.D. conducted molecular modeling. C.K., J.P.N., M.D.W., and M.A.D. assembled figures. M.D.W. and M.A.D. conceived the project. C.K., T.N., C.Z.B., Y.A., J.J., S.M., C.L.C., C.B., M.D.W., and M.A.D. performed data analysis. C.K., M.D.W., and M.A.D. wrote the manuscript. All authors reviewed and edited the paper.

\section{REFERENCES}

Campbell BB, Light N, Fabrizio D, Zatzman M, Fuligni F, de Borja R, Davidson S, Edwards M, Elvin JA, Hodel $\mathrm{KP}$, et al. 2017. Comprehensive analysis of hypermutation in human cancer. Cell 171: 1042-1056.e1010. doi:10.1016/j.cell.2017.09.048

Cerami E, Gao J, Dogrusoz U, Gross BE, Sumer SO, Aksoy BA, Jacobsen A, Byrne CJ, Heuer ML, Larsson E, et al. 2012. The cBio cancer genomics portal: an open platform for exploring multidimensional cancer genomics data: figure 1. Cancer Discov 2: 401-404. doi:10.1158/2159-8290.CD-12-0095

Chang MT, Asthana S, Gao SP, Lee BH, Chapman JS, Kandoth C, Gao J, Socci ND, Solit DB, Olshen AB, et al. 2016. Identifying recurrent mutations in cancer reveals widespread lineage diversity and mutational specificity. Nat Biotechnol 34: 155-163. doi:10.1038/nbt.3391

Cocco E, Scaltriti M, Drilon A. 2018. NTRK fusion-positive cancers and TRK inhibitor therapy. Nat Rev Clin Oncol 15: 731-747. doi:10.1038/s41571-018-0113-0

Cocco E, Schram AM, Kulick A, Misale S, Won HH, Yaeger R, Razavi P, Ptashkin R, Hechtman JF, Toska E, et al. 2019. Resistance to TRK inhibition mediated by convergent MAPK pathway activation. Nat Med 25: 1422 1427. doi:10.1038/s41591-019-0542-z

Davare MA, Vellore NA, Wagner JP, Eide CA, Goodman JR, Drilon A, Deininger MW, O'Hare T, Druker BJ. 2015. Structural insight into selectivity and resistance profiles of ROS1 tyrosine kinase inhibitors. Proc Natl Acad Sci 112: E5381-E5390. doi:10.1073/pnas.1515281112

Degasperi A, Amarante TD, Czarnecki J, Shooter S, Zou X, Glodzik D, Morganella S, Nanda AS, Badja C, Koh $G$, et al. 2020. A practical framework and online tool for mutational signature analyses show intertissue variation and driver dependencies. Nat Cancer 1: 249-263. doi:10.1038/s43018-020-0027-5

Doebele RC, Drilon A, Paz-Ares L, Siena S, Shaw AT, Farago AF, Blakely CM, Seto T, Cho BC, Tosi D, et al. 2020. Entrectinib in patients with advanced or metastatic NTRK fusion-positive solid tumours: integrated analysis of three phase 1-2 trials. Lancet Oncol 21: 271-282. doi:10.1016/S1470-2045(19)30691-6

Drilon A, Li G, Dogan S, Gounder M, Shen R, Arcila M, Wang L, Hyman DM, Hechtman J, Wei G, et al. $2016 a$. What hides behind the MASC: clinical response and acquired resistance to entrectinib after ETV6-NTRK3 
identification in a mammary analogue secretory carcinoma (MASC). Ann Oncol 27: 920-926. doi:10.1093/ annonc/mdw042

Drilon A, Somwar R, Wagner JP, Vellore NA, Eide CA, Zabriskie MS, Arcila ME, Hechtman JF, Wang L, Smith RS, et al. 2016b. A novel crizotinib-resistant solvent-front mutation responsive to cabozantinib therapy in a patient with ROS1-rearranged lung cancer. Clin Cancer Res 22: 2351-2358. doi:10.1158/1078-0432.CCR15-2013

Drilon A, Nagasubramanian R, Blake JF, Ku N, Tuch BB, Ebata K, Smith S, Lauriault V, Kolakowski GR, Brandhuber BJ, et al. 2017. A next-generation TRK kinase inhibitor overcomes acquired resistance to prior TRK kinase inhibition in patients with TRK fusion-positive solid tumors. Cancer Discov 7: 963-972. doi:10 .1158/2159-8290.CD-17-0507

Drilon A, Laetsch TW, Kummar S, DuBois SG, Lassen UN, Demetri GD, Nathenson M, Doebele RC, Farago AF, Pappo AS, et al. 2018a. Efficacy of larotrectinib in TRK fusion-positive cancers in adults and children. N Engl J Med 378: 731-739. doi:10.1056/NEJMoa1714448

Drilon A, Ou SI, Cho BC, Kim DW, Lee J, Lin JJ, Zhu WW, Ahn MJ, Camidge DR, Nguyen J, et al. 2018b. Repotrectinib (TPX-0005) is a next-generation ROS1/TRK/ALK inhibitor that potently inhibits ROS1/TRK/ ALK solvent- front mutations. Cancer Discov 8: 1227-1236. doi:10.1158/2159-8290.CD-18-0484

Gao J, Aksoy BA, Dogrusoz U, Dresdner G, Gross B, Sumer SO, Sun Y, Jacobsen A, Sinha R, Larsson E, et al. 2013. Integrative analysis of complex cancer genomics and clinical profiles using the cBioPortal. Sci Signal 6: pl1. doi:10.1126/scisignal.6273er1

Gao J, Chang MT, Johnsen HC, Gao SP, Sylvester BE, Sumer SO, Zhang H, Solit DB, Taylor BS, Schultz N, et al. 2017. 3D clusters of somatic mutations in cancer reveal numerous rare mutations as functional targets. Genome Med 9: 4. doi:10.1186/s13073-016-0393-x

Hemming ML, Nathenson MJ, Lin J-R, Mei S, Du Z, Malik K, Marino-Enriquez A, Jagannathan JP, Sorger PK, Bertagnolli $\mathrm{M}$, et al. 2020. Response and mechanisms of resistance to larotrectinib and selitrectinib in metastatic undifferentiated sarcoma harboring oncogenic fusion of NTRK1. JCO Precis Oncol 4: 79-90. doi:10 1200/po.19.00287

Hiemenz MC, Ostrow DG, Busse TM, Buckley J, Maglinte DT, Bootwalla M, Done J, Ji J, Raca G, Ryutov A, et al. 2018. OncoKids: a comprehensive next-generation sequencing panel for pediatric malignancies. $J$ Mol Diagn 20: 765-776. doi:10.1016/j.jmoldx.2018.06.009

Hong DS, DuBois SG, Kummar S, Farago AF, Albert CM, Rohrberg KS, van Tilburg CM, Nagasubramanian R, Berlin JD, Federman N, et al. 2020. Larotrectinib in patients with TRK fusion-positive solid tumours: a pooled analysis of three phase 1/2 clinical trials. Lancet Oncol 21: 531-540. doi:10.1016/S1470-2045 (19)30856-3

Huang EJ, Reichardt LF. 2003. Trk receptors: roles in neuronal signal transduction. Annu Rev Biochem 72: 609642. doi:10.1146/annurev.biochem.72.121801.161629

Johnson BE, Mazor T, Hong C, Barnes M, Aihara K, Mclean CY, Fouse SD, Yamamoto S, Ueda H, Tatsuno K, et al. 2014. Mutational analysis reveals the origin and therapy-driven evolution of recurrent glioma. Science 343: 189-193. doi:10.1126/science.1239947

Katayama R, Gong B, Togashi N, Miyamoto M, Kiga M, Iwasaki S, Kamai Y, Tominaga Y, Takeda Y, Kagoshima $Y$, et al. 2019. The new-generation selective ROS1/NTRK inhibitor DS-6051 b overcomes crizotinib resistant ROS1-G2032R mutation in preclinical models. Nat Commun 10: 3604. doi:10.1038/s41467-019-11496-z

Noeparast A, Giron P, Noor A, Bahadur Shahi R, De Brakeleer S, Eggermont C, Vandenplas H, Boeckx B, Lambrechts D, De Greve J, et al. 2019. CRAF mutations in lung cancer can be oncogenic and predict sensitivity to combined type II RAF and MEK inhibition. Oncogene 38: 5933-5941. doi:10.1038/s41388-019-0866-7

Pandit B, Sarkozy A, Pennacchio LA, Carta C, Oishi K, Martinelli S, Pogna EA, Schackwitz W, Ustaszewska A, Landstrom A, et al. 2007. Gain-of-function RAF1 mutations cause Noonan and LEOPARD syndromes with hypertrophic cardiomyopathy. Nat Genet 39: 1007-1012. doi:10.1038/ng2073

Pettersen EF, Goddard TD, Huang CC, Couch GS, Greenblatt DM, Meng EC, Ferrin TE. 2004. UCSF Chimeraa visualization system for exploratory research and analysis. J Comput Chem 25: 1605-1612. doi:10.1002/ jcc. 20084

Russo M, Misale S, Wei G, Siravegna G, Crisafulli G, Lazzari L, Corti G, Rospo G, Novara L, Mussolin B, et al. 2016. Acquired resistance to the TRK inhibitor entrectinib in colorectal cancer. Cancer Discov 6: 36-44. doi:10.1158/2159-8290.CD-15-0940

Somwar R, Hofmann NE, Smith B, Odintsov I, Vojnic M, Linkov I, Tam A, Khodos I, Mattar MS, de Stanchina E, et al. 2020. NTRK kinase domain mutations in cancer variably impact sensitivity to type I and type II inhibitors. Commun Biol 3: 776. doi:10.1038/s42003-020-01508-w

Torre M, Vasudevaraja V, Serrano J, DeLorenzo M, Malinowski S, Blandin AF, Pages M, Ligon AH, Dong F, Meredith DM, et al. 2020. Molecular and clinicopathologic features of gliomas harboring NTRK fusions. Acta Neuropathol Commun 8: 107. doi:10.1186/s40478-020-00980-z

Warmuth M, Kim S, Gu XJ, Xia G, Adrian F. 2007. Ba/F3 cells and their use in kinase drug discovery. Curr Opin Oncol 19: 55-60. doi:10.1097/CCO.0b013e328011a25f 
Xu J, Reumers J, Couceiro JR, De Smet F, Gallardo R, Rudyak S, Cornelis A, Rozenski J, Zwolinska A, Marine J-C, et al. 2011. Gain of function of mutant p53 by coaggregation with multiple tumor suppressors. Nat Chem Biol 7: 285-295. doi:10.1038/nchembio.546

Ziegler DS, Wong M, Mayoh C, Kumar A, Tsoli M, Mould E, Tyrrell V, Khuong-Quang DA, Pinese M, Gayevskiy $V$, et al. 2018. Brief Report: potent clinical and radiological response to larotrectinib in TRK fusion-driven high-grade glioma. Br J Cancer 119: 693-696. doi:10.1038/s41416-018-0251-2 


\section{COLD SPRING HARBOR Molecular Case Studies}

\section{Mechanisms of targeted therapy resistance in a pediatric glioma driven by ETV6-NTRK3 fusion}

Clare Keddy, Tanaya Neff, Jianya Huan, et al.

Cold Spring Harb Mol Case Stud 2021, 7: a006109 originally published online August 24, 2021

Access the most recent version at doi: $10.1101 / \mathrm{mcs} .0006109$
Supplementary http://molecularcasestudies.cshlp.org/content/suppl/2021/09/08/mcs.a006109.D Material C1

References This article cites 30 articles, 8 of which can be accessed free at: http://molecularcasestudies.cshlp.org/content/7/5/a006109.full.html\#ref-list-1

License This article is distributed under the terms of the Creative Commons Attribution-NonCommercial License, which permits reuse and redistribution, except for commercial purposes, provided that the original author and source are credited.

Email Alerting Receive free email alerts when new articles cite this article - sign up in the box at the Service top right corner of the article or click here. 